\title{
Assessing the Prevalence of Dental Injuries while Playing Sports as well as Awareness of Preventive Measures in Schoolchildren of Amritsar City: A Cross-sectional Study
}

\author{
Savreen Kaur ${ }^{1}$, Supreet Kaur ${ }^{2}$, Gunmeen Sadana ${ }^{3}$, Teena Gupta ${ }^{4}$, Rashu Grover ${ }^{5}$
}

\begin{abstract}
Aims and Objectives: To assess the prevalence of dental injuries while playing sports as well as awareness of preventive measures in school children of Amritsar city.

Materials and methods: A study was conducted on 500 children aged between 6 and 16 years with a mean age of 13 years. A questionnaire was planned to evaluate the prevalence of dental injuries while playing sports and their awareness regarding the use of mouthguards while playing. The answering group consisted of $67.2 \%$ of male and $32.8 \%$ of female children.

Results: Two-hundred and ninety (58\%) children had participated in collision contact sports, $138(28 \%)$ in impact contact sports, $58(11 \%)$ in moderately strenuous contact, $9(2 \%)$ in strenuous contact, and $5(1 \%)$ in non- strenuous contact sports. While 149 (29.8\%) experienced facial injury, the rest 51 (10.2\%) had bone breakage injury while playing sports. Almost $31 \%$ of the participants thought wrapping in cotton or cloth is the best way to carry the avulsed tooth to the dental clinic, whereas only $14 \%$ had the knowledge that milk is the best-suited medium. The majority of the children [316 (63\%)] were not aware that it was possible to reimplant the teeth. About $30 \%$ thought that teeth can be reimplanted after washing them with water and then immediately going to the dentist to reimplant them, and $25 \%$ thought that wrapping in paper and visiting the dentist immediately or the next day to reimplant the teeth. Out of the total participants, 160 (32\%) used mouthguards and 340 $(68 \%)$ had never used mouthguards, whereas $58.8 \%$ were aware of preventive measures and $41.2 \%$ had no such knowledge.

Conclusion: The prevalence of dental trauma in three schools of Amritsar district was $20.20 \%$, so education on preventive measures should be given to the trainers and trainees to prevent dental injuries while playing sports. The use of mouthguards should be made mandatory for school kids while playing.
\end{abstract}

Keywords: Awareness, Mouthguards, Sports injury.

AMEl's Current Trends in Diagnosis \& Treatment (2021): 10.5005/jp-journals-10055-0123

\section{INTRODUCTION}

A physically active lifestyle comprising of sporting and recreational activities is essential for all age-groups. Perhaps the probabilities of suffering injuries in the orofacial area have increased while playing sports, and dental injuries are the commonly occurring type of orofacial injury. Other causative factors that account for trauma are fall and road traffic accidents. Diverse researches have concluded that sports-related injuries have been reported for 10 to $36 \%$ of injuries in all causes. Dental injuries are generally found among children in the age between 8 years and 15 years, although recently an increasing trend has been seen among patients older than 18. ${ }^{1,2}$

In school, children actively participate in outdoor activities. Although these activities indicate the growth and development of the child, careless acts, imbalance, and discoordinated actions increase the risk of injuries. Traumatic injuries in the dental aspect in the primary dentition are likely to be the sequelae affecting the permanent teeth, and irregularity has been estimated to occur in $25-69 \%$ of cases. ${ }^{3}$ The most frequent tooth injuries affecting the primary teeth were limited to the supporting tissues, that is, subluxation, avulsion, or intrusive luxation. In the permanent dentition, uncomplicated fracture of the tooth crown was by far the most frequent finding. ${ }^{4}$

The term "tooth avulsion" implies a situation where, as a result of trauma, a tooth has been removed from its socket. When the tooth is out of the socket, the cells of the pulp and the periodontal ligament start to weaken. This is owing to the lack of
1,2,5 Department of Pedodontics and Preventive Dentistry, Sri Guru Ram Das Institute of Dental Sciences and Research, Amritsar, Punjab, India

${ }^{3,4}$ Department of Pedodontics, Sri Guru Ram Das Institute of Dental Sciences and Research, Amritsar, Punjab, India

Corresponding Author: Supreet Kaur, Department of Pedodontics and Preventive Dentistry, Sri Guru Ram Das Institute of Dental Sciences and Research, Amritsar, Punjab, India, Phone: +91 6283832394, e-mail: supreetkaur0638@gmail.com

How to cite this article: Kaur S, Kaur S, Sadana G, et al. Assessing the Prevalence of Dental Injuries while Playing Sports as well as Awareness of Preventive Measures in Schoolchildren of Amritsar City: A Crosssectional Study. AMEl's Curr Trends Diagn Treat 2021;5(1):31-35.

Source of support: Nil

Conflict of interest: None

blood supply to the cells and environmental factors (e.g., drying or bacterial contamination). The prognosis of an avulsed tooth is basically reliant on the condition of the periodontal ligament cells at reimplantation. Successful instant management of the avulsed tooth is thus primarily concerned with reimplanting the tooth with the viability of these cells undamaged. ${ }^{5}$

Growing awareness of these issues has motivated the development of protective devices, such as helmets and mouthguards, which are used in sports to minimize the chances and severity of 
sports-related traumatic injuries to the head, face, and mouth. In certain sports (e.g., martial arts), such measures have been in standard use for many decades. ${ }^{6}$ Sports dentistry had its commencement in the 1980s, and it encompasses the injury-prone dentition and proficiency in the immediate management of dental injuries. With the increasing trend of contact sports and encouragement to take part at an early age, the part of the dental profession in relation to the prevention of dental and other orofacial sporting injuries has become more essential. So as to scrutinize, children, coaches, parents, and members of the dental community should be aware of how individuals, who participate in sports, are at risk for dental trauma. ${ }^{7}$

This cross-sectional study thus aimed to assess the prevalence of dental injuries while playing sports as well as awareness of preventive measures in schoolchildren of the Amritsar city.

\section{Materials and Methods}

This cross-sectional study was performed in a group of 500 children in the age range from 6 to 16 years in three schools of the Amritsar district after taking permission from the school authorities. The children belonging to the answering group played different sports, that is, football, basketball, badminton, cricket, skating, boxing, tennis, table tennis, swimming, and gymnastics. All children who were in the age range between 16 years and below and able to understand English or Hindi language were included in the study conducted from November 2019 to January 2020.

A structured, guided questionnaire was formulated in closedended form for data collection by the author and coauthors involved in the study. The questionnaire was approved by a group of experts, and thus, a pilot study was conducted through the distribution of hard copies of proforma among 20 children participating in different sports activities for their understanding of the language. The questionnaire enclosed 15 points, including the type of sports that children play, time period since when they had been practicing that sport, and whether any facial and breaking of bone injuries occurred, particularly mobility of teeth and fracture of teeth, while participating in sports activities. Children were inquired of whether they knew that it was possible to reimplant the avulsed tooth and also the extraoral time within which it is feasible to reimplant the tooth. Children were asked how they would store or carry a tooth that is out of socket to their dentist. They were asked that if they know that when teeth are proclined, then the chances of tooth injury are more. They were also asked about the awareness of any preventive measures to avoid injury during sports. Participants were inquired of whether they use mouthguard while playing sports and if not, mention the reasons for not using it, and whether they consider mouthguard can prevent dental injury or not. The data thus collected were statistically analyzed.

\section{Results}

A total of 500 children from three schools participated in the study. Among the total participants, 336 (67.2\%) were males and 164 $(32.8 \%)$ were females. Their age range was from 6 to 16 years with a mean age of 13 years.

Children participate in various sports comprised of football, basketball, badminton, cricket, skating, boxing, tennis, table tennis, swimming, and gymnastics. The distribution of participants in various sports is shown in Table 1 (Fig. 1).

- Among the total participants, 290 (58\%) had participated in collision contact sports, $138(28 \%)$ in impact contact sports,
$58(11 \%)$ in moderately strenuous contact, $9(2 \%)$ in strenuous contact, and $5(1 \%)$ in non-strenuous contact sports.

- Out of the total participants, the majority had no injury while playing sports, that is, 300 (60\%). While 149 (29.8\%) experienced facial injury, the rest $51(10.2 \%)$ had a history of fracture while playing sports Table 2 (Fig. 2).

- Various types of storage medium can be used for avulsed teeth for the reimplantation of the teeth. Almost 31\% of the participants thought wrapping in cotton or cloth is the best way to carry avulsed tooth to the dentist, while only $14 \%$ knew that milk is the most suitable medium Table 3 (Fig. 3).

- Out of the total participants, the majority of the children [316 (63\%)] were not familiar with the fact that it was possible to reimplant the teeth. About 30\% thought that teeth can be reimplanted after washing them with water, while $25 \%$ thought that wrapping it in paper and then visiting the dentist to reimplant the teeth. While $24 \%$ thought keeping the tooth in any medium and then visiting the dentist, 19\% thought tooth can be reimplanted immediately Table 4 (Fig. 4).

- Out of the total participants, 294 (58.8\%) children were aware of preventive measures to avoid tooth injury, whereas 206 (41.20\%) were unaware of any preventive measures to avoid tooth injury Table 5 (Fig. 5).

Table 1: Distribution of participants in various contact sports

\begin{tabular}{lcc}
\hline Type of contact & Frequency & Percent \\
\hline Collision contact & 290 & 58.00 \\
Impact contact & 138 & 27.60 \\
Strenuous contact & 9 & 1.80 \\
Moderately strenuous contact & 58 & 11.60 \\
Non-strenuous contact & 5 & 1.00 \\
\hline
\end{tabular}

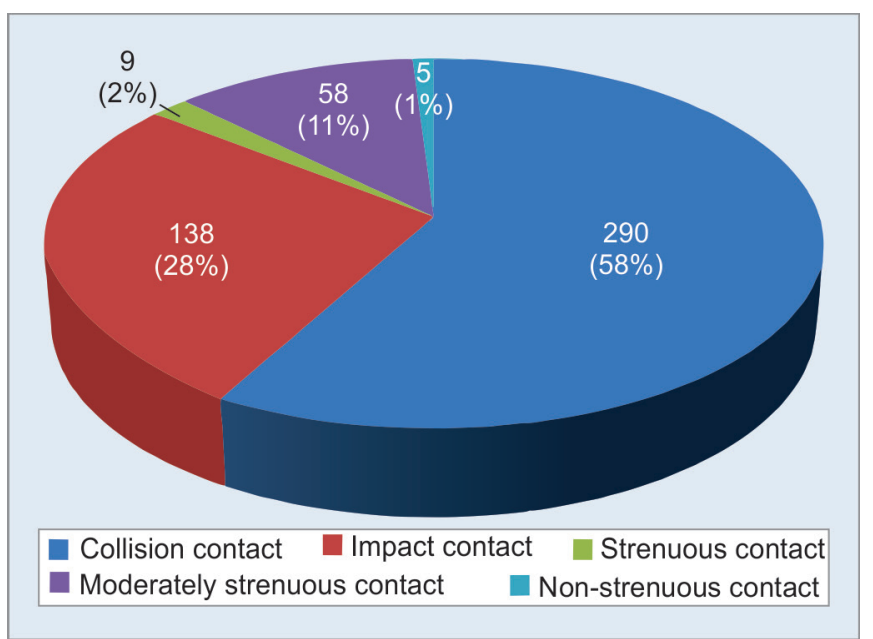

Fig. 1: Distribution of participants in various contact sports

Table 2: Types of injuries sustained

\begin{tabular}{lcl}
\hline Type of injury & Frequency & Percent \\
\hline Facial injury & 149 & 29.80 \\
Bone break & 51 & 10.20 \\
No injury & 300 & 60.00 \\
\hline
\end{tabular}


- Out of the total participants, $160(32 \%)$ used mouthguard while playing, whereas 340 (68\%) had never used mouthguards Table 6 (Fig. 6).

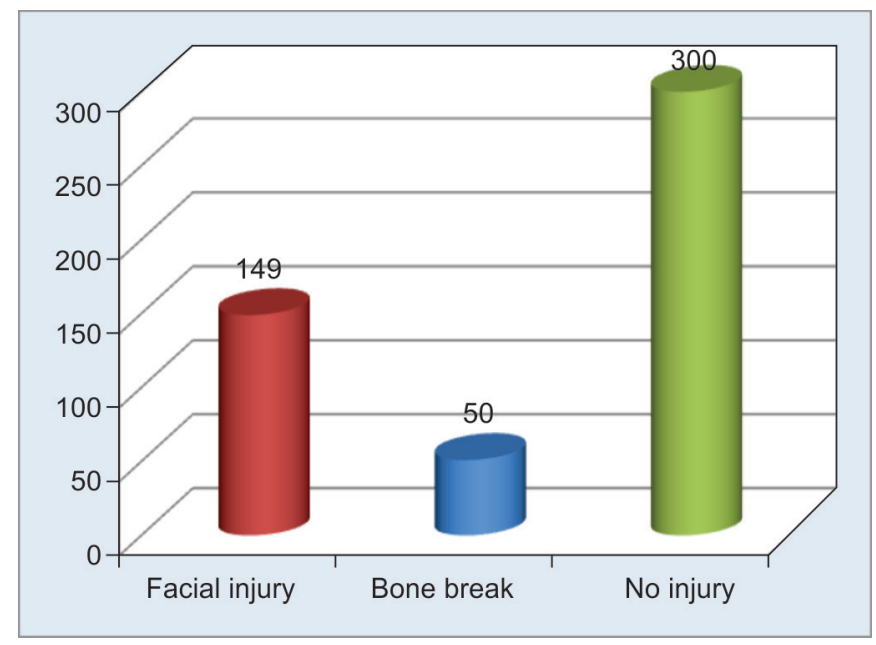

Fig. 2: Types of injuries sustained

Table 3: Medium used to carry the avulsed teeth

\begin{tabular}{lcl}
\hline Medium to carry avulsed teeth & Frequency & Percent \\
\hline In your mouth/saliva & 82 & 16.40 \\
Water & 128 & 25.60 \\
Wrapped in cloth or cotton & 156 & 31.20 \\
Coconut water & 61 & 12.20 \\
Milk & 73 & 14.60 \\
\hline
\end{tabular}

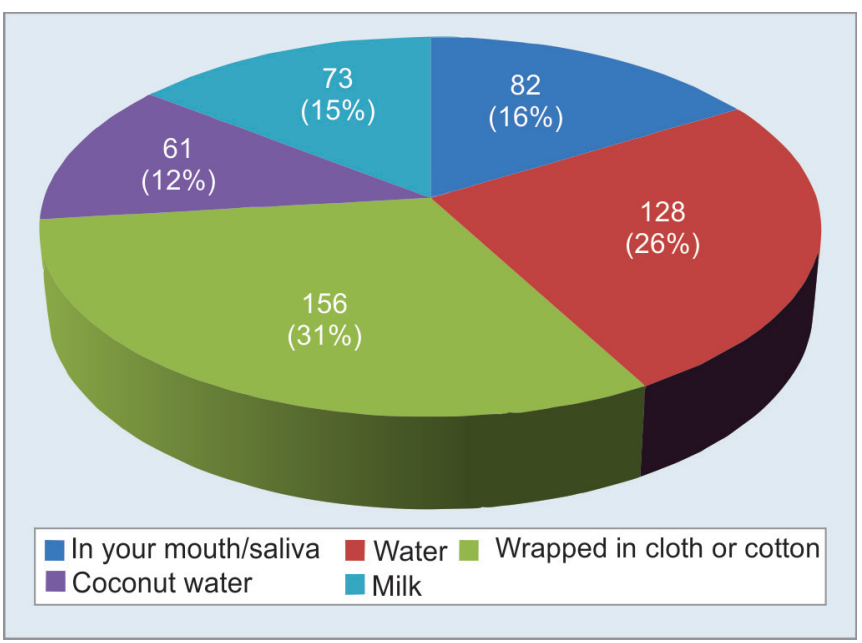

Fig. 3: Medium used to carry avulsed tooth

Table 4: Time duration for teeth to be reimplanted

\begin{tabular}{lcl}
\hline \multicolumn{3}{c}{ Broken tooth handling } \\
\hline Timespan to put tooth back & Frequency & Percent \\
\hline Immediately & 96 & 19.20 \\
After washing it with water & 152 & 30.40 \\
Keeping in any medium and visiting dentist & 124 & 24.80 \\
Wrapping in paper and visiting dentist & 128 & 25.60 \\
\hline
\end{tabular}

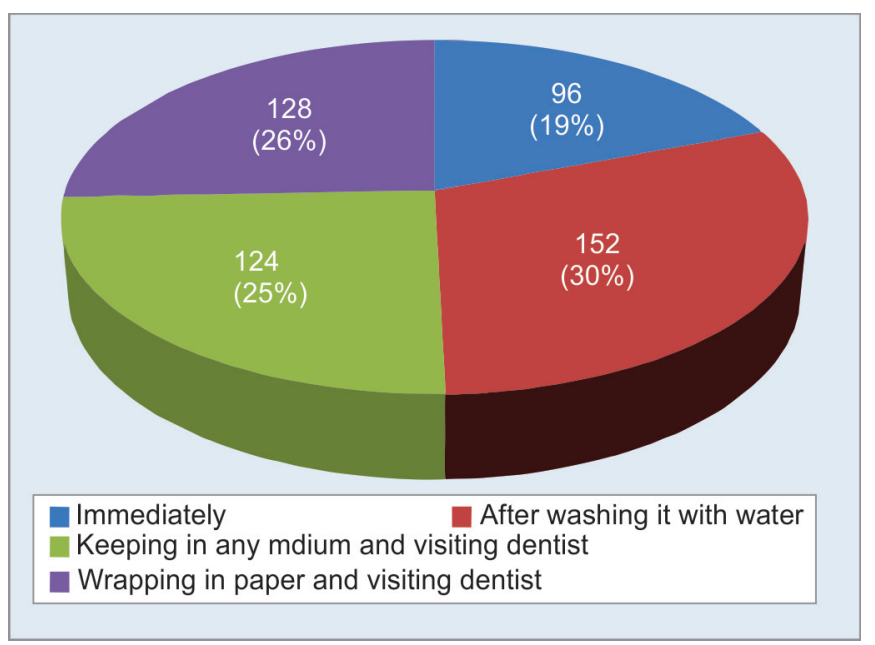

Fig. 4: Time span to put avulsed tooth back in the oral cavity

Table 5: Knowledge on preventive measures to avoid tooth injury

\begin{tabular}{lll}
\hline Knowledge on preventive measures & Frequency & Percent \\
\hline Yes & 294 & 58.80 \\
No & 206 & 41.20 \\
\hline
\end{tabular}

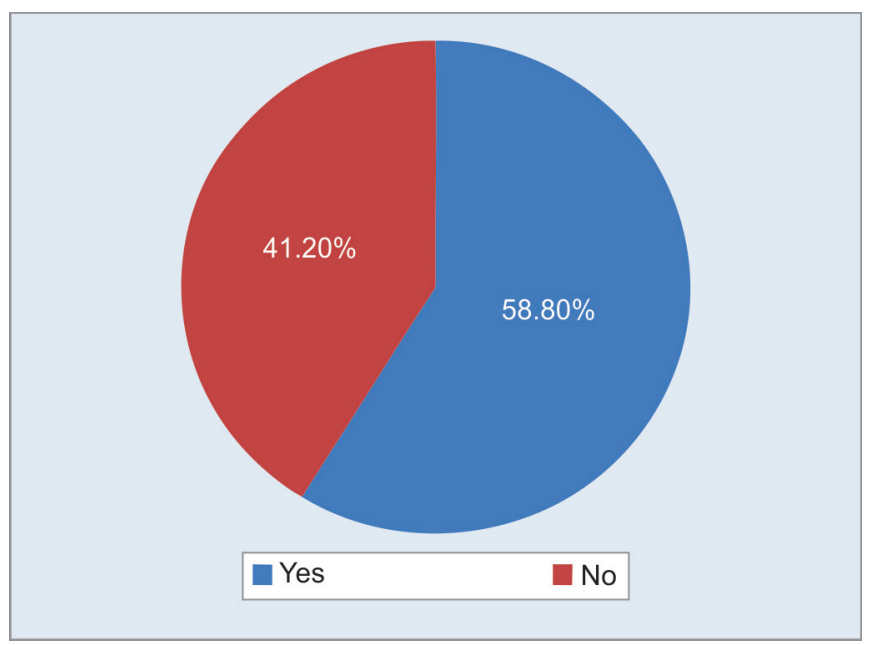

Fig. 5: Knowledge on preventive measure to avoid tooth injury

Table 6: Mouthguard use

\begin{tabular}{lll}
\hline Mouthguard use & Frequency & Percent \\
\hline Yes & 160 & 32.00 \\
No & 340 & 68.00 \\
\hline
\end{tabular}

\section{Discussion}

School-going children especially and adolescents involve in frivolous and competitive sports activities for physical as well as psychological well-being. ${ }^{7}$ A physically active lifestyle is essential for all. Reasons to participate in sports and physical activity are several, such as recreation, competition, socialization, and maintenance of fitness and health.

The World Health Organization theme in the year 2002 says "Move for health," which emphasizes the role of physical activity in 


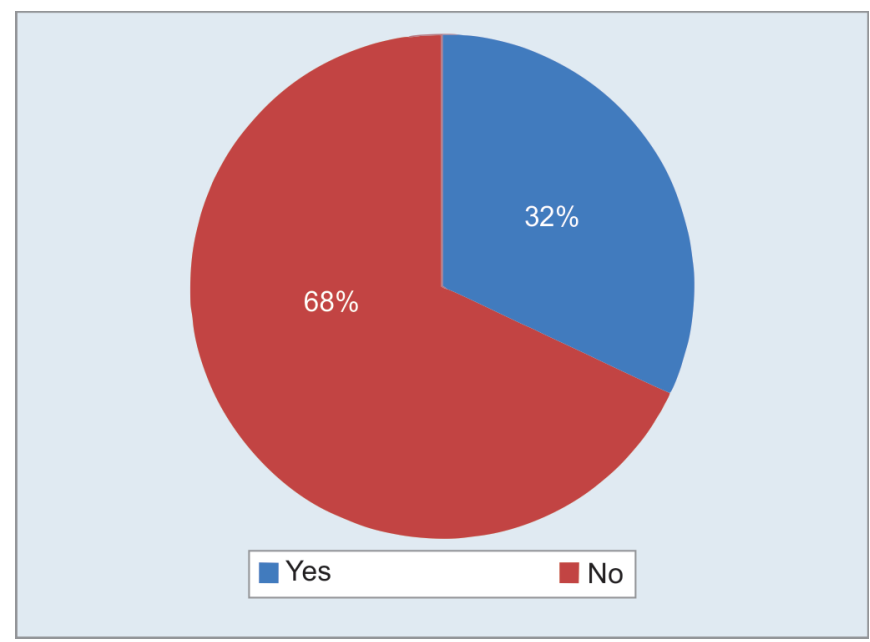

Fig. 6: Mouthguard use while playing sports

the healthy lifestyle of an entity. For this phase of physical activities, injuries to the face are one of the risks related to it. Trauma to both primary and permanent dentition continues to be a common dental problem ${ }^{3}$

Importance should not only be constrained to oral diseases but encompasses the prevention of dental injuries. All anticipatory measures should be based on appropriate knowledge. Prevention of dental injuries should, therefore, be based on reliable data relating to incidence, diagnosis, and causes. ${ }^{8}$

Prevalence rates of $5-12 \%$ in dental injuries due to trauma are found in children in the age range of 6-12 years in the Middle East. In a study by Patel et al., it was found that from certain developed countries, the prevalence of dental traumatic injuries is on the increase, ranging from 16 to $40 \%$ and 4 to $33 \%$ among 6-year-old and 12-14-year-old children, respectively. ${ }^{9}$

The present study reveals $58 \%$ of students are involved in collision contact sports. About $29.80 \%$ of students had experienced orofacial injuries while playing sports. This result agrees with the studies done by Tulunoglu and Ozbeck and Persic et al., where 22.3 and $20.4 \%$ of the participants reported to have experienced oral injuries, respectively. . $^{10,11}$

The prevalence of dental trauma in three schools of the Amritsar district was $20.20 \%$, which is in accordance with the prevalence of traumatic dental injuries occurring worldwide, which ranges from 6 to $37 \%$. In the present study, $79.80 \%$ of them were sustained during playing sports. The prime purpose of conducting study in these three urban sector schools was that it includes middle and upper middle-class students who certainly have more access to sports and recreational activities. Sports-related dental injuries are high in percentage among all types of traumatic injuries across the world.

The status of knowledge among the individuals who were questioned about reimplantation of the avulsed tooth was another part of our study evaluating the level of information on emergency management of dental trauma. In the present study, 184 among 500 (36.80\%) had the knowledge that it was feasible to reimplant the avulsed tooth. Another study by Ersin et al. showed only $34.7 \%$ of individuals had the knowledge about reimplantation of the teeth. ${ }^{12} \mathrm{~A}$ study by Goswami et al. showed that 263 out of 450 (58.4\%) individuals had known that it was possible to reimplant the avulsed tooth. ${ }^{7}$

Most of the participants (30.40\%) in our study were not aware much about the time duration in which the avulsed tooth could be reimplanted. Only $19.20 \%$ think that the time span to put the tooth back can be immediate; $30.40 \%$ are of the view after washing it with water; $24.80 \%$ were aware that avulsed tooth should be kept in any medium and visiting dentist; and $25.60 \%$ think that tooth should be wrapped in paper and then visiting the dentist.

Other studies have shown that $31.6 \%$ of individuals had not much knowledge about the time duration in which the avulsed tooth could be reimplanted, and only $8.3 \%$ had known that avulsed tooth should be carried in a liquid medium (milk, water, and saliva). ${ }^{12,13}$ In our study, in the majority of the children, 156 (31.20\%) out of 500 thinks that avulsed tooth should be wrapped in cloth or cotton, while $25.60 \%$ knew water, (16.40\%) saliva, (14.60\%), milk, and (12.20\%) coconut water as a storage medium; this is because of increasing concern and knowledge about dental health.

Another factor associated with anterior tooth trauma is the proclination of teeth. Nearly, $56.4 \%$ did not know that if teeth are proclined, then the probability of tooth injury is more. Children with inadequate lip coverage have five times more susceptibility to injuries. Insufficient lip coverage has been shown as an important risk factor and was found to be a significant determinant for dental injuries. The risk of oral injuries during sports and exercise can be reduced substantially by using mouthguards. ${ }^{13}$ Mouthguards offer protection by separating the cheeks and lips from the teeth, making users less prone to soft tissue laceration and preventing opposing arches from traumatic contact, and these protective devices provide a robust, protective surface to distribute and dissipate transmitted forces on impact. ${ }^{14}$

Despite medical and dental groups emphasizing the use of professionally fitted headgears and mouthguards to minimize head, facial, and dental injury, it has not gained much popularity among children in India. Regarding the usage and awareness of mouthguard, $32 \%$ of children in the present study were effectively using it. However, $58.80 \%$ of children have knowledge on preventive measures, and $41.20 \%$ were unaware of any such measures to avoid tooth injury. Athletes not wearing mouthguards are also widespread in other developed countries. Various studies have revealed that though the participants were aware of the importance of mouthguards, very few were really using them. ${ }^{15}$

These findings support that not just the mere knowledge only on mouthguards use does not guarantee its utilization. Furthermore, children and coaches should be well-versed that the physical impacts of having injuries go beyond the expenses of purchasing and the hassle of wearing a mouthguard. The most familiar reason why children $(58.80 \%)$ did not put mouthguard to use was that their coaches did not emphasize about the use of the mouthguard.

The present study had shown that price is not the only one factor for not wearing a mouthguard, but lack of contemplation on the significance of mouthguard in preventing oral injury is the main reason for not wearing a mouthguard.

\section{Conclusion}

- Sports-related dental injuries have comprised of high percentage among all types of traumatic injuries worldwide.

- Prevalence of dental trauma in three schools of the Amritsar district was $20.20 \%$.

- Thirty-seven percent of children had known that it was feasible to reimplant the avulsed tooth.

- There was a shear lack of knowledge regarding the storage medium used for carrying the avulsed tooth. Majority of the 
children (31.20\%) thinks that avulsed tooth should be wrapped in cloth or cotton.

- Majority of the participants had the knowledge about the role of mouthguards in the prevention of injury, but most of them did not use mouthguards because of a lack of encouragement from the coaches.

- Pediatric dentists in collaboration with pediatricians should guide parents for the prevention and management of trauma due to sports-related dental injuries.

- An effort can be made to diminish the prevalence of traumatic injuries by taking into consideration the use of intraoral and extraoral devices that protect the face and teeth from trauma.

- Educational programs are conducted where the children and their parents are given information concerning the prevention and treatment aspects of this commonly occurring condition.

\section{References}

1. Hecova $H$, Tzigkounakis V, Merglova V, Netolicky J. A retrospective study of 889 injured permanent teeth. Dent Traumatol 2010;26(6): 466-475. DOI: 10.1111/j.1600-9657.2010.00924.x.

2. Bahr R, Holme I. Risk factors for sports injuries: a methodological approach. Br J Sports Med 2003;37(5):384-392. DOI: 10.1136/ bjsm.37.5.384.

3. Gojanur S, Yeluri R, Munshi AK. Prevalence and etiology of traumatic injuries to the anterior teeth among 5 to 8 years old school children in Mathura city, India: an epidemiological study. Int J Clin Pediatr Dent 2015;8(3):172. DOI: 10.5005/jp-journals-10005-1308.

4. Borssén E, Holm AK. Treatment of traumatic dental injuries in a cohort of 16-year-olds in northern Sweden. Endod Dent Traumatol 2000;16:276-281. DOI: 10.1034/j.1600-9657.2000.016006276.x.

5. Donaldson M, Kinirons MJ. Factors affecting the time of onset of resorption in avulsed and replanted incisor teeth in children.
Dent Traumatol 2001;17(5):205-209. DOI: 10.1034/j.16009657.2001.170503.x.

6. Levin L, Friedlander LD, Geiger SB. Dental and oral trauma and mouthguard use during sport activities in Israel. Dent Traumatol 2003;19(5):237-242. DOI: 10.1034/j.1600-9657.2003.00196.x.

7. Goswami M, Kumar P, Bhushan U. Evaluation of knowledge, awareness, and occurrence of dental injuries in participant children during sports in New Delhi: a pilot study. Int J Clin Pediatr Dent 2017;10(4):373-378. DOI: 10.5005/jp-journals-10005-1468.

8. Ankola AV, Hebbal M, Sharma R, Nayak SS. Traumatic dental injuries in primary school children of South India-a report from districtwide oral health survey. Dent traumatol 2013;29(2):134-138. DOI: 10.1111/j.1600-9657.2012.01139.x.

9. Patel MC, Sujan SG. The prevalence of traumatic dental injuries to permanent anterior teeth and its relation with predisposing risk factors among 8-13 years school children of Vadodara city: an epidemiological study. J Indian Soc Pedod Prev Dent 2012;30(2):151. DOI: 10.4103/0970-4388.99992.

10. Tulunoglu I, Ozbek M. Oral trauma, mouthguard awareness, and use in two contact sports in Turkey. Dent Traumatol 2006;22(5):242-246. DOI: 10.1111/j.1600-9657.2006.00386.x.

11. Persic R, Pohl Y, Filippi A. Dental squash injuries - a survey among players and coaches in Switzerland, Germany and France. Dent Traumatol 2006;22(5):231-236. DOI: 10.1111/j.1600-9657.2006.00379.x.

12. Ersin N, Ertugrul F, Oncag O, Menderes M, Uzel I. Evaluation of high risk university students' knowledge about dental trauma and emergency procedures. J Int Dent Med Res 2013;6(3):100-104.

13. Woodmansey KF. Athletic mouth guards prevent orofacial injuries: a review. Gen Dent 1999;47(1):64-69. DOI: 10.1080/07448481. 1997.9936880.

14. ADA Council on Access, Prevention and Interprofessional Relations; ADA Council on Scientific Affairs. Using mouthguards to reduce the incidence and severity of sports related oral injuries. J Am Dent Assoc 2006;137(12):1712-1720. DOI: 10.14219/jada.archive.2006.0118.

15. Dale RA. Dentoalveolar trauma. Emerg Med Clin N Am 2000;18(3): 521-553. DOI: 10.1016/s0733-8627(05)70141-3. 